\title{
A minimally invasive model data passing interface for integrating legacy environmental system models
}

\author{
A.Q. Dozier, O. David, M. Arabi, W. Lloyd, Y. Zhang \\ Colorado State University, Fort Collins, CO, USA
}

\begin{abstract}
This paper presents an approach to model integration utilizing the Model Data Passing Interface (MODPI). The approach provides fine-grained, multidirectional feedbacks between legacy environmental system models through read and write access to relevant model data during simulation using a bidirectional, event-based, publish-subscribe system. MODPI only requires commented directives in the original code and an XML linkage file with an optional custom data conversion module. Automated code generation, compilation, and execution reduce the programming burden on the modeler. Case study results indicated that MODPI required less code modifications within each model code base both before and after automated code generation, outperforming a baseline subroutine approach. Performance overhead for MODPI was minimal for the use case, offering speedup in some cases through parallel execution. MODPI is much less invasive than other techniques, encouraging adoption by the modeling community and improving maintainability and reusability of integrated model code.
\end{abstract}

Keywords: Framework invasiveness, Integrated Assessment and Modeling,

Email address: andre.dozier@rams.colostate.edu (A.Q. Dozier)

Preprint submitted to Environmental Modelling \& Software

December 31, 2015

(C) 2016. This manuscript version is made available under the Elsevier user license http://www.elsevier.com/open-access/userlicense/1.0/ 
Integrated Environmental Modeling, Inter-Process Communication

1 Data and Software Availability

2 Software developed for the purposes of this paper are open-source and 3 publicly available. The implementation of MODPI presented in the paper is

4 found at https://bitbucket.org/adozier/fortmodpi (2.1 MB), and the 5 implementation of events is found at https://bitbucket.org/adozier/ fortevents (11 KB). A summary of the packages is found at https://www. erams.com/resources/Platform/MaaS/Model_Integration. This software requires an implementation of the Message Passing Interface (MPI) or Ze9 roMQ. Models (3 MB), performance test results (370 kB), a shell script that 10 reproduces the results $(2 \mathrm{kB})$ in this paper are found at https://erams. com/resources/Platform/MaaS/Model_Integration.

12

\section{Introduction}

Model integration frameworks, or environmental modeling frameworks, allow a plug-and-play methodology in connecting submodels of an environmental system to enhance model representation of the overall system. However, framework invasiveness restricts reuse and maintenance of frameworkdependent models (Donatelli and Rizzoli, 2008; Lloyd et al., 2011). Thus, instead of using the frameworks, many environmental system model developers incorporate transplanted, and often outdated, submodels of other disciplines into their codes (Laniak et al., 2013). Although modeling frameworks have been used to integrate such models across disciplines, model developers often maintain the model code base separate from its support of the framework. 
Lloyd et al. (2011) defines framework invasiveness as "the quantity of dependencies between model code and a modeling framework". Modeling frameworks that aim at minimizing imposed dependencies on a legacy model improve maintainability and reuse (Lloyd et al., 2011). Historically, modeling frameworks that attempt to be less invasive have focused on coarse-grained interaction between "components" or submodels of a larger model (Donatelli and Rizzoli, 2008; Lloyd et al., 2011). Finer-grained feedback schemes that exchange data from within a component or submodel have previously taken more invasive approaches or require extensive computer programming expertise (Becker and Schuttrumpf, 2011). We argue that the amount of work required within a legacy model for integration with another model or support of a framework interface is another obstacle to framework adoption. Thus, we define invasiveness here to be the dependencies within each model on either the integration platform or other models, and the amount of work required within each model for integration or implementation of a framework interface.

Attempts to incorporate multidirectional feedback between legacy models include iterative, subroutine, and inter-process communication approaches that are discussed in detail in the next section. To remain minimally invasive, inter-process communication techniques are the most promising approaches as demonstrated by Becker and Schuttrumpf (2011) in making a closed-source model compliant with the OpenMI standard (Moore and Tindall, 2005) within a timestep loop. Inter-process communication techniques have previously required too much programming knowledge for most modelers. Thus, there still remains a need for a minimally invasive, fine-grained, 
generic model integration interface that does not require such extensive programming expertise (Laniak et al., 2013).

The goal of developing the Model Data Passing Interface (MODPI) is to facilitate and abstract the legacy model integration process to reduce framework invasiveness while minimizing programming knowledge requirements. Objectives of this study include:

1. Develop an abstracted interface for minimally invasive model integration that simplifies complex interactions between legacy models and modeling platforms of disparate disciplines,

2. Automate code generation of MODPI-compatible wrappers for legacy models to support ease-of-use, and

3. Evaluate invasiveness and performance of MODPI as compared to other approaches.

To accomplish these objectives, a publish-subscribe concept is combined with inter-process communication to provide external processes read and write access to any relevant state variable within a legacy model during its execution. A framework is built that automates wrapper generation, and a case study serves to benchmark MODPI against another common approach to the same problem.

\section{Background and Related Work}

8 Many researchers have previously addressed specific model integration challenges, and some have even developed generic interfaces for model integration. However, no generic interface exists for fine-grained, multidirectional 
feedbacks that preserves the individuality and maintainability of legacy models. Implementing interfaces for existing frameworks requires significant work within the model, and often requires addition of code dependencies on the framework.

This section identifies previous studies that have integrated legacy models to support fine-grained, multidirectional feedbacks, which is the primary functional requirement for the integration studies we summarize here. Finegrained feedbacks refer to linkages of internal (and relevant) data or calculations between multiple models that cannot be represented by one model as a whole, but are required to represent a particular process more accurately. Multidirectional feedbacks refer to data or calculations within one model that depend on another model and vice versa. When the need for such feedbacks between models arises, there are various implementation considerations such as 1) implicit versus explicit numerical solution techniques, 2) passing data via subroutines or put/get calls, 3) hardware mechanism for communication, and 4) single or multiple executable approaches (Valcke et al., 2012). The following framework design targets for MODPI are used to qualitatively assess the different approaches:

\section{Minimally invasive}

2. Minimal interface requirements

3. Interoperable across languages and platforms

4. Links closed-source models

5. Reconciles data structure differences

6. Performance overhead is minimal

Interoperability and data structure reconciliation are functional requirements 
for specific model integration tasks, which also may be true for linking closedsource models and performance overhead in some cases, but not for a generic model integration interface.

Framework design targets are prioritized to make the interface more acceptable to a diverse modeling community that individually maintains or uses large legacy models. We argue that the first two targets, invasive changes within a model code base and difficult or extensive interface requirements, represent the largest factors that inhibit maintenance and reuse of integrated modeling systems (Lloyd et al., 2011). The design target for minimal interface requirements is aimed at reducing the amount of programming work and knowledge required to be able to implement MODPI for a model. Since modelers are often limited by an unfamiliarity with advanced programming techniques to improve interoperability or link closed-source models, minimizing difficult code changes and refactoring requirements may provide a path to encourage adoption and reuse of model integration frameworks. Ensuring interoperability of languages, platforms, and linkages with closed-source models would also broaden the applicability of an integration platform within an increasingly diverse community of modelers (Laniak et al., 2013).

\subsection{Implicit versus Explicit Approaches}

Both implicit and explicit solution approaches have advantages and disadvantages. Although solving equations explicitly may intuitively seem numerically faster, implicit approaches may utilize assumptions to solve much more efficiently without sacrificing too much accuracy (Balaji, 2012). Within hydrology, several approaches based on successive approximations allow models to be run separately, maintaining model individuality in partial fulfillment 
of Target 1 (Fredericks et al., 1998; Ibanez et al., 2014). Lagrangian relaxation techniques are systematic implicit numerical methods that allow for parallel execution of submodels Dozier (2012). Because implicit approaches utilize original forms of equations, model individuality may be more easily attained than explicit approaches addressing Targets 1, 3, 4 and 5. However, most explicit solutions can improve geophysical model integration through guaranteeing numerical solutions for feasible inputs (Dozier, 2012; Balaji, 2012).

\subsection{Subroutines versus Producer-Consumer Approaches}

In model integration, data can be passed through subroutine arguments or through an exchanging mechanism such as a buffer that handles producers and consumers through put/get calls (i.e., publishers and subscribers). These are distinguished from hardware communication mechanisms because both subroutine arguments and buffers could potentially utilize memory, hard disk, or network communications, although there are typical implementations.

Implementing a subroutine approach often entails decomposing submodels into smaller components: initialization, run or update, and finalization (Argent, 2004). For example, the Basic Model Interface (BMI) specifies initialize and finalize methods in addition to an update function that is used to advance a model or component to the next timestep while data is passed by name via interface subroutines between updates (Peckham et al., 2013). Kim et al. (2008) integrated a surface water model (SWAT) with a subsurface water model (MODFLOW) by splitting MODFLOW up into its sub-components, calling the computational component of MODFLOW within the groundwater module of SWAT, and compiling the models to- 
gether. In the case of the Object Modeling System (OMS), data is exchanged between multiple models during their simulation through separate (and potentially parallel) execution of model subcomponents based on data availability (David et al., 2013). OMS avoids framework dependence through annotations and custom wrappers in partial fulfillment of Target 1, but requires decomposing models into smaller components for fine-grained analysis (Lloyd et al., 2011; David et al., 2013).

In most cases with put/get approaches, data has previously been passed through specific inter-process communication (IPC) or framework dependencies within the original model code base (Valcke et al., 2012; Armstrong et al., 2009). Dozier (2012) showed that event constructs can be utilized to decouple models from the integrated modeling system while using the subroutine approach, which could be extended to the producer-consumer approach. Although the Earth System Modeling Framework (ESMF), the Common Component Architecture (CCA), and Bespoke Framework Generator (BFG2) primarily utilize subroutine-based data passing techniques, they allow subcomponents to use producer-consumer types of data interactions through shared memory or some form of IPC (Allan et al., 2006; Armstrong et al., 1999, 2006; Collins et al., 2005; Hill et al., 2004; Larson et al., 2004; Lefantzi et al., 2003; Armstrong et al., 2009; Valcke et al., 2012). In cases where the framework removes required IPC dependencies from the model, only coarse-grained interactions between model subcomponents are allowed, requiring the model to be broken into smaller components for fine-grained analysis (Hill et al., 2004; Collins et al., 2005). 


\subsection{Hardware Communication Mechanisms}

Mechanisms for communicating data between models primarily include memory, hard drives, and network communications. For typical hardware speeds, Dean (2009) describes that in general a disk seek is 100,000 times slower than a reference to main memory, and reading $1 \mathrm{MB}$ from disk is 80 times slower than from memory. To communicate data through slower hardware such as hard drives or network infrastructure may prove to have unacceptable overhead, although most model integration tasks require little data exchange compared to model computations. For example, automating the model integration in the cases of Fredericks et al. (1998) and Ibanez et al. (2014) would have little framework overhead regardless of hard drive reads and writes because of extremely long model runtimes relative to the small data passing requirements. In designing a framework, though, care should be taken to minimize overhead because it may be applied to a large variety of models with different runtimes and data passing requirements.

\subsection{Single versus Multiple Executable Approaches}

Single executable applications typically require language interoperability, which has been enhanced through tools like Babel (Dahlgren et al., 2012) as used by Peckham et al. (2013). IPC techniques are well-known multiprocessing methods for establishing interoperability by being platform-, framework, and language-independent (Magnoni, 2015). As discussed in Section 2.2, prior frameworks have either required models to implement IPC mechanisms or major refactoring to break models into subcomponents for fine-grained interactions in multiple executables, trading off invasiveness with the number of interface requirements. 


\subsection{Discussion of Previous Work}

With regard to Targets 1 and 2, when a model requires significantly refactoring for model integration purposes or dependencies on the integrated modeling system (i.e., either other models or a model integration framework), it often no longer retains individuality or separability from the framework. That is, we argue that invasiveness and excessive interface requirements discourage adoption, maintainability, and reusability of the integrated model code base by splitting legacy models into two code bases, one that implements the interface and one that does not. These two model code bases can sometimes be merged into one when interface requirements are minimally invasive as in the case of BMI (Peckham et al., 2013).

Laniak et al. (2013) express a need for interoperability (Target 3) between different disciplinary models and between integrated environmental modeling frameworks that are implemented with different programming languages, compilers, and platforms (Matott et al., 2009). IPC techniques address this need for interoperability between models with varying architectural, platform, or license dependencies, but has previously required invasive approaches or required extensive programming expertise (Targets 1 and 2). Compiling multiple models together into one executable requires models be interoperable either directly, or at least utilize a language interoperability tool such as Babel (Dahlgren et al., 2012). In our experience with re-implementing the approach by Yuan et al. (2011) discussed in Section 5, interoperability of original model code was also compromised when compiling legacy models together because of conflicting compiler flags, and overlapping global variable names or file handles. Also, maintaining the state of the submodel 
between timestep advancements required additional debugging and refactoring. Baart et al. (2014) improved interoperability of models implementing the Basic Modeling Interface (BMI) through an IPC technique.

Although component-based modeling may be easier to understand and program, Target 4 with regard to linking closed-source models prohibits excessive refactoring of model code, which would need to be done by the model developer. Dozier (2012) showed that event constructs can be utilized to decouple model codes, maintain model individuality, and allow plug-and-play with different models, even closed-source models. Becker and Schuttrumpf (2011) improved interoperability (Target 3) of this approach by implementing the Open Modelling Interface (OpenMI) standard (Moore and Tindall, 2005) for a closed-source model through event constructs and remote procedure calls. However, the extent to which a geophysical scientist is capable of programming IPC in a similar manner is extremely limited, consequently negatively impacting its potential acceptance in the broader modeling community.

Data structures need to be reconciled when compiling models together or performing inter-process communication, but are facilitated by input and output file formats when passing data via the hard drive (Target 5). Although exchanging data via subroutines is one of the most utilized approaches for fine-grained integration, legacy model developers do not often maintain support of model integration interfaces, thus downplaying advantages of plugand-play model integration (Donatelli and Rizzoli, 2008; Lloyd et al., 2011).

Performance overhead (Target 6) must be taken into consideration especially in cases when data must be passed between models at a fine-grained 
resolution in time or space relative to the number of computations performed by each model. Hard drives and networks communicate data between models more slowly than main memory, but overhead may be minimal when computations outweigh data exchange requirements.

\subsection{A New Interface}

A generic interface for decoupling computations and disciplinary models using IPC (to address interoperability, Target 3) without requiring extensive legacy code refactoring (Target 1, 2, and 4) or unacceptable performance overhead (Target 6) while generically allowing for data structure reconciliation (Target 5) still remains to be developed. MODPI is introduced here to address this need by attempting to satisfy each of the framework design targets through use of an event-based publish-subscribe system with a message broker (Salas, 2012). Although there are still limitations to the use of MODPI and required work on the part of the modeler, MODPI represents a significant advancement towards satisfying each of the framework design targets. Fig. 1 displays a schematic of the form of model integration presented by MODPI.

Section 3 describes the interface, its components, requirements, and optional features. Section 4 describes the implementation of the MODPI integration platform and choice of IPC mechanism for interoperability purposes. Section 5 illustrates the use of MODPI with a case study application linking two legacy models, DayCent and HYDRUS. Section 6 summarizes the results of the case study by presenting measures of required code modifications and performance of the integrated modeling system. Section 7 presents discussions of benefits, limitations, advantages, and disadvantages of MODPI 


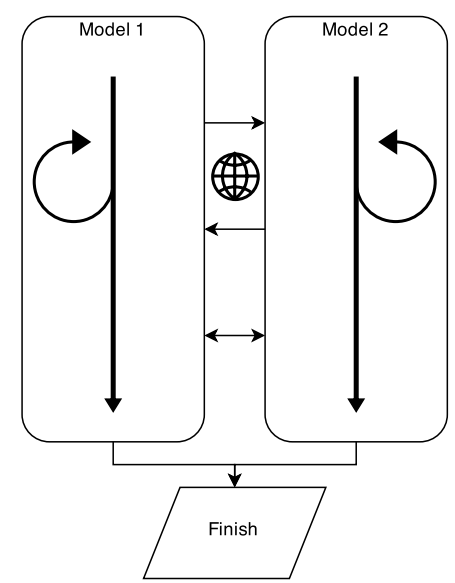

Figure 1: Selected implementation of MODPI links model coupling equations either implicitly or explicitly, and passes data through publisher-subscriber interactions across separate processes. Depending on the inter-process communication mechanism and machine setup, data can be passed through either hard disk, shared memory, or network communications.

and its use for further different studies. Section 8 concludes with a discussion about the benefits of MODPI, its design considerations, and results of the case study application, while Section 9 presents potential future work to improve this study and future implementations of MODPI.

\section{The Model Data Passing Interface}

MODPI provides read and write access to any relevant variable by name within a legacy model during simulation without legacy code refactoring through use of event constructs and an automated IPC message broker. MODPI formalizes, adapts, and expands the approach used by Becker and Schuttrumpf (2011) to generically and automatically apply IPC to any model, open-source or closed-source. A legacy model must implement the following elements to become MODPI-compatible: 


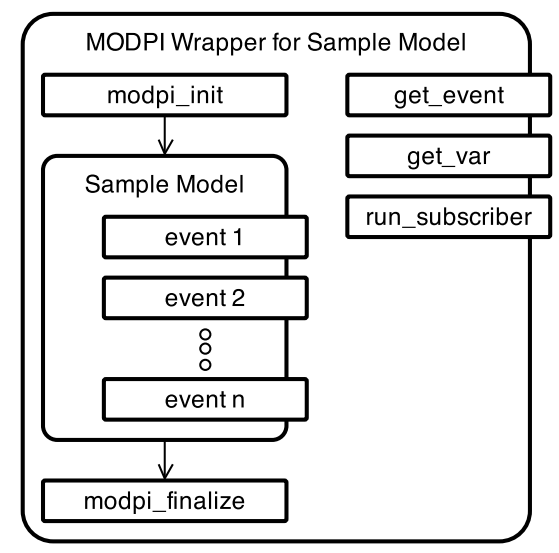

Figure 2: Schematic of a wrapper program for a sample model that implements the Model Data Passing Interface (MODPI) using its three interface functions get_event, get_var, and run_subscriber, and runs the MODPI-compatible sample model after initializing and before finalizing.

1. Legacy model as a library with MODPI directives as code comments

2. Generated wrapper program with optional customization

3. XML linkage file

These essential elements of MODPI are discussed in the following three subsections and shown in Fig. 2 for a sample model. The MODPI integration platform refers to a controller that automates code generation (for event constructs and wrapper programs) and runs the integrated modeling system. The component that exchanges data between models during simulation using IPC is called the MODPI Broker.

\subsection{Legacy Model as a Library with MODPI Directives}

A legacy model that implements MODPI must be accessible to the MODPI integration platform as a library, and must contain MODPI directives. Prior 
to compilation, the MODPI integration platform requires MODPI directives to generate the event constructs, but during compilation and runtime, it requires the actual event constructs to access model data. Thus, MODPI allows for the capability of wrapping closed-source models when generated event constructs are kept within the pre-compiled libraries delivered to model users, after which MODPI directives are no longer required to stay in the source code. The requirements for MODPI compatibility are very minimal changes to a legacy model code base. The model does not need to be decomposed into initialization, body, and finalization subcomponents, but remains entirely intact. The hardest legacy models to refactor into multiple components are poorly modularized codes that utilize a lot of global data. These very common, yet poorly modularized models provide good use cases for MODPI since localized model data requires additional event data constructs where global data does not. Although being able to access both local and global data within a model allows MODPI flexibility, the model developer (in the case of closed-source models) or the one integrating models (in either closed-source or open-source models) should still take care to encapsulate or hide data from the model integration interface that should remain "private" in accordance with good practice. Local data is hidden from MODPI by not providing it as event data. Global data is hidden at compilation by not exporting certain variables to the library.

Directives have the following syntax directly after a comment mark ("!" in FORTRAN or "//" in C) to add an import statement and to invoke an event, respectively:

! MODPI\$ use

! MODPI\$ event <event_name $>(\langle$ type > <intent $\rangle\langle$ variable_name $\rangle[\langle$ size $\rangle], \ldots$ ) 
MODPI directives are placed within the legacy model code as code comments to support automatic generation of event, or callback, constructs to allow any number of subscribers to access local or global data at various points during model execution. Automatic generation of event constructs from directives is a functional requirement for integration platforms implementing the Model Data Passing Interface to simplify programming responsibilities for the modeler, and to render MODPI less invasive. Automation is especially useful in low-level languages such as FORTRAN and C due to the lack of language-supported event constructs. Event constructs may remain in the model without affecting its normal execution either apart from or within MODPI linkages.

Using MODPI directives, the automated event generator determines where to place use or import statements, when to fire events, and the data to provide as event data. If all variables declared locally are to be added as event data, the keyword all locals is used in addition to any variable names used. Types are declared using either $\mathrm{C}$ or FORTRAN data type syntax (e.g., int and integer are interchangeable). If the event invocation lies within a $\mathrm{C}$ source file, the type should include an asterisk symbol ("*") when a variable is declared as a pointer within the surrounding context. Intent of event data is an optional parameter that is declared using the following FORTRAN syntax:

1. intent (in): variable is passed by value

2. intent (inout): variable is passed by reference (or as a pointer)

3. intent (out): variable is an output parameter only

Intent is used primarily to ensure that parameter types are passed by value 
when declared as a constant, instead of being passed as a pointer, the default in FORTRAN. If a variable is an array, its dimensions are specified using square brackets after the variable name similar to a $\mathrm{C}$ declaration, and includes constant numbers or other variable names. The modeler tasked with implementing MODPI for a model will want to ensure that data that is meant to be read-only should be passed as value with intent(in).

The entire MODPI-compatible model is called as a subroutine within the compiled library. The model must not exit in a nonstandard manner (e.g., through using the stop keyword in FORTRAN) because the IPC mechanism needs to be finalized. An optional consideration for making a model compatible with MODPI is to ensure that any redundant calculations are switched off. As in the case study in Section 5, a detailed, physically-based model replaces the soil-water submodel of the biogeochemical model, which is switched off to avoid calculations that are overridden anyways.

\subsection{Generated Wrapper Program with Optional Customization}

A wrapper program implements MODPI for a legacy model by using the legacy model as a library. The MODPI integration platform generates the wrapper program in addition to the event constructs given MODPI directives, the XML linkage file, and an optional custom, user-written module. Any custom actions written in the module are not overwritten when code generation is performed, but are rather subscribed to events in the model based on subscriptions within the XML linkage file. The wrapper program implements through interface functions, initializes and finalizes MODPI, and 
runs the legacy model. ${ }^{1}$ Fig. 2 shows each component and control flow of a wrapper program.

When initializing, the MODPI Broker first obtains user-specified model connection information from the XML linkage file. Then, it subscribes event handlers to event constructs, and initializes the IPC mechanism. After executing the model, the MODPI Broker finalizes the IPC mechanism. The wrapper program informs the MODPI Broker when to initialize and finalize by performing the following three tasks consecutively:

1. Initialize MODPI by executing modpi_init

2. Run the model by executing its main subroutine

3. Finalize MODPI by executing modpi_finalize

The wrapper program also implements three interface functions:

1. get_event: retrieves events within the model

2. get_var: retrieves data within the model

3. run_subscriber: executes custom subscribers to the model

These three functions allow the MODPI Integration Platform to retrieve model events and data, and to run custom subscribers during runtime based on specifications within the XML linkage file described in the next section.

Custom subscribers perform any calculations necessary to convert and manipulate data for any connection with another model (or modeling system) without requiring code changes to the original legacy code base. During runtime, the MODPI Broker automatically subscribes any custom data

\footnotetext{
${ }^{1} \mathrm{~A}$ detailed discussion of each interface function and other attributes of wrapper programs is found at https://bitbucket.org/adozier/fortmodpi/wiki/wrapper_detail
} 
converter to events specified by the XML linkage file, allowing the modeler to easily toggle specific data conversions. Additionally, custom subscribers are compiled into the wrapper program for a model, not into the model itself, thus decoupling model integration customization from the model to aid maintainability of the integrated modeling system with future updates to the model code base. Still, custom subscriber modules must be updated when there is a change in names or locations of events and event data in addition to when a data structure is altered. When custom subscribers are changed, the automated code generator needs to be executed again to generate a run_subscriber routine that runs new custom subscribers. Although custom data conversions might require more maintenance than if the MODPI Integration Platform performed generic data conversions, MODPI remains minimally invasive because it does not require certain data structures within MODPI-compatible model code bases. Since customized model integration actions are performed outside of the model library through events and event data, there are minimal maintenance requirements compared to approaches that add framework dependencies to the model code.

\section{3. $X M L$ Linkage File}

An XML file specifies data to exchange between MODPI-compatible models. Its structure provides the list of connected models, the data that is to be exchanged, the data conversions that are to take place, and the intermediate data required for data conversions. When building the XML file, a modeler must be able to understand that data can be passed from one model to another during its execution, but is not required to know how to program message passing into any code. 
At runtime, the MODPI Broker requires the XML file to be named "modpi.xml" and found in the working directories for each of the models. Prior to runtime, the MODPI integration platform copies and renames the original XML file into each of the working directories automatically. The XML file path is not passed to MODPI as a command line argument so that some legacy models do not attempt parsing the file path incorrectly, as MODPI is designed to be minimally invasive. Examples and detailed descriptions of the XML file are found in the repository. ${ }^{2}$

\section{The MODPI Integration Platform}

This section describes the MODPI integration platform developed and used in this paper. Fig. 3 displays the steps required to implement MODPI for any model, where preliminary model changes and MODPI directives allow a wrapper program to be generated. Linkage to another model is provided through the MODPI XML linkage file. MODPI is highly interoperable between programming languages and platforms, being tested in FORTRAN, $\mathrm{C}$, and $\mathrm{C}++$, and preliminarily tested in Java and MATLAB script on both Ubuntu and Windows operating systems. MODPI has also been tested to straddle different virtual machines, different physical machines, and Ubuntu and Windows operating systems, running one model in Ubuntu and the other in Windows.

The subsections below describe concepts and features of three components of the MODPI integration platform:

\footnotetext{
${ }^{2}$ The XML file definition is found at https://bitbucket.org/adozier/fortmodpi/ wiki/xml
} 


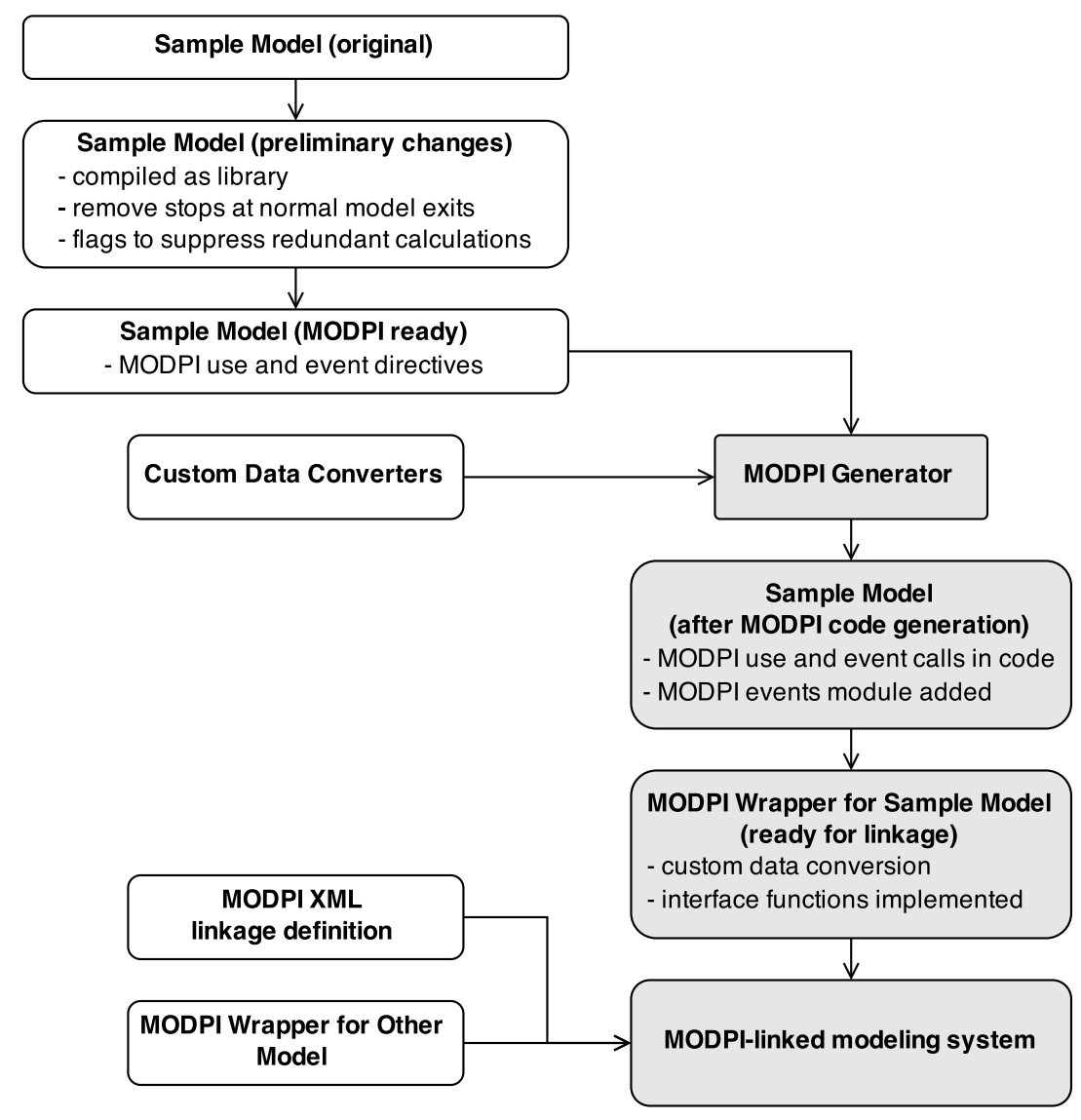

Figure 3: A flow chart displaying steps to implement MODPI for a generic model. A MODPI wrapper code generator within the MODPI integration platform called "modpi_generate.py" automates the generation of events, event data, and MODPI interface functions. Gray elements highlight the generated components of the integrated modeling system. 


\section{Event constructs}

2. The MODPI Broker

3. The automated code generator

Two generic libraries, one for event constructs and one for the MODPI integration platform, are used within this paper to demonstrate implementation of MODPI. Concrete code references and detailed descriptions for the XML linkage file, event constructs, wrapper programs, automatic code generation and execution, and tutorials on using the MODPI integration platform can be found at the online MODPI repository. ${ }^{3}$

\subsection{Event Constructs}

The term "events" instead of "callbacks" is used here because the MODPI integration platform utilizes new object-oriented features of the Fortran 2003 standard. Events refer to a class of objects that run any number of subscribed subroutines to allow for customization without changing the control flow of the original code or adding many logic statements. Event invocations are placed in locations within the code of a model or component where something of interest happens or is about to happen.

Subscribers are subroutines that handle an event to perform customized calculations with model data at the location of the event during model execution. When invoked, an event executes each subscribing subroutine while passing data associated with the event as an argument. When no subscribers

\footnotetext{
${ }^{3}$ The open-source and publicly available repositories for the implementation of events and the MODPI integration platform is found at https://bitbucket.org/adozier/ fortevents and https://bitbucket.org/adozier/fortmodpi, respectively.
} 

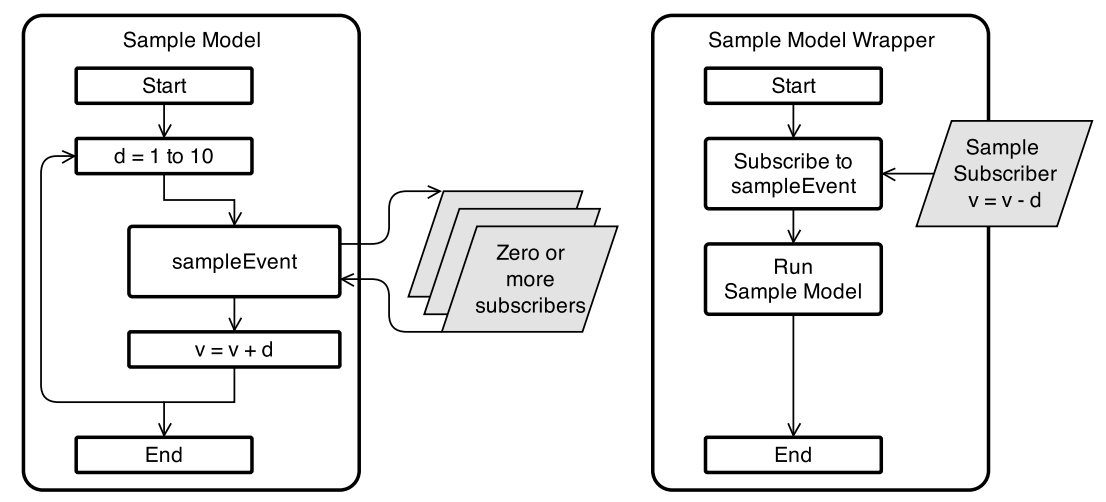

Figure 4: Schematic of a sample model that adds the numbers 1 through 10, and a wrapper that subscribes to the event within the data. This subscriber simply negates the addition within the sample model.

exist, the firing method of the event simply exits, consequently leaving the original flow of the model unchanged.

Fig. 4 illustrates events and subscribers for a sample model that adds the numbers one through ten. The wrapper subscribes to events within the sample model, and thus has access to local data that the event provides in addition to any globally exported variables within the library. In this sample case, the subscriber negates the calculations made by the sample model prior to the calculations being performed. If the sample model is executed by itself, it runs normally without needing recompilation.

Events add value to MODPI by decoupling the model from the integration platform while still allowing access to model state, whether local or global, during execution. To be MODPI-compatible, a model needs to fire events during its simulation, and its source code remains closed if desired when the model is compiled as a library and appropriate variables are exported. The 
legacy model code base is executed and maintained separately from its support of any specific integration with another model and separate from the integration platform. Other approaches, such as compiling models together or implementing an invasive model integration framework, create two separate code bases that need to be individually maintained in order to keep the integration intact with future versions of the model, whereas MODPI allows merging these two branches of code into one, reducing maintenance requirements. We argue that the use of events in MODPI will result in a higher probability of framework adoption by the broader modeling community because events reduce framework invasiveness by preserving model control flow and individuality.

A user of MODPI is not required to manually implement events within the legacy model, because the interface only requires MODPI directives within the comments of the legacy model code base. The MODPI integration platform then automatically generates the event constructs within the legacy model, without loss of generality since the event constructs are very generic structures. However, so that the reader understands the event construct, this section defines events and subscribers to reinforce why they render MODPI less invasive.

\subsection{The MODPI Broker}

The MODPI integration platform performs data exchange between models through a construct called the MODPI Broker, which uses IPC to exchange data between MODPI-compatible models and also automatically subscribes custom data converters to events within the models. The modeler looking to implement MODPI for a legacy model does not need to build the 
MODPI Broker. Fig. 3 displays steps required to implement MODPI for a generic model, and distinguishes automated steps. This section discusses the features, functionality, and dependencies of the various MODPI Broker components.

The MODPI Broker developed for use in this paper is implemented in FORTRAN, which is currently the most commonly used language for legacy geophysical models. This broker implementation effectively links models built in FORTRAN, $\mathrm{C}$, or $\mathrm{C}++$. It utilizes either the Message Passing Interface, MPI (The MPI Forum, 1993), or ZeroMQ (Hintjens, 2013) as the selected forms of IPC since they are well-developed standards, have widespread community support, and have proved to be very interoperable approaches. Interaction schemes implemented in this version of the broker are MPI_Send and MPI_Recv (and similar calls for ZeroMQ) with limited broadcasting support. Due to the dependency of the MODPI Broker on MPI, the number of times a variable is sent from one model must be matched with the number of times a variable is received in the other model, and vice versa. Thus, if timestep loops encompass events where MODPI exchanges data, the number of timesteps must be equivalent across models. Otherwise, execution will hang at the end of the simulation.

Fig. 5 illustrates the connection between the MODPI Broker, a MODPIcompatible model, and the wrapper program. The MODPI Broker subscribes to events retrieved by get_event and performs message passing between other models running as separate processes to exchange data retrieved through the get_var subroutine. The broker automatically subscribes and executes custom data converters to events within the model using the user- 


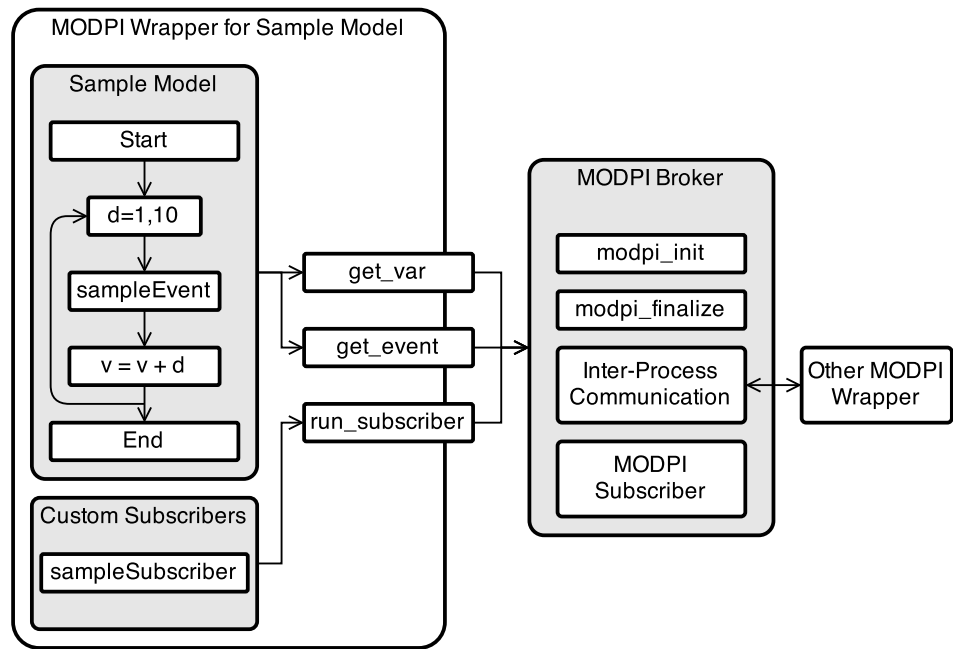

Figure 5: A MODPI wrapper for a sample model to illustrate how the MODPI Broker connects models through the use of the interface functions get_var, get_event, and run_subscriber and IPC.

supplied run_subscriber subroutine. The implementation and interfaces for these subroutines are discussed in more detail in the online repository. ${ }^{4}$

\subsection{Automated Code Generation, Compilation, and Execution}

MODPI requires that events, event data, and necessary wrapper components be automatically generated from MODPI directives within the comments of the source code. Section 3.1 describes the syntax and usage of the MODPI directives, which render MODPI less invasive and potentially easier to use by novice programmers. To automatically implement MODPI for a MODPI-compatible model, the modeler places MODPI directives within comments of the original legacy model code, writes any necessary custom

\footnotetext{
${ }^{4}$ See footnote 3
} 
data converters, potentially creates an XML linkage file to declare intermediate variables, and runs the automatic generation tool. Code generation and automated compilation is performed for each model separately or for all models in the integrated modeling system simultaneously.

Automatic detection of model source files, custom data converters, and library directories is achieved through use of the recommended directory structure shown in Fig. 6. After automated code generation, MODPI directives within the original model source code are replaced with import statements and calls to subroutines that fire events. A generated events module declares event constructs and associated event data, and is compiled into the original model source code. The generated wrapper program discussed in Section 3.2 includes the custom data converter and two other files that implement the three interface functions, initializes the MODPI Broker, runs the model, and finalizes the MODPI Broker.

Closed-source models are provided as a library, without original source files. If customization of the generated MODPI-compatible wrapper program is desired, the generated events module should be provided to users of the model. In the case study discussed in Section 5, DayCent acts as a closedsource model to demonstrate an implementation of MODPI for closed-source models.

The code generator automatically attempts to compile the generated wrapper program and associated model. Automated code compilation is meant for models and wrapper programs written in any combination of FORTRAN, C, or $\mathrm{C}++$. Compiled libraries, modules, and executable files are automatically placed in their respective directory locations according to the 


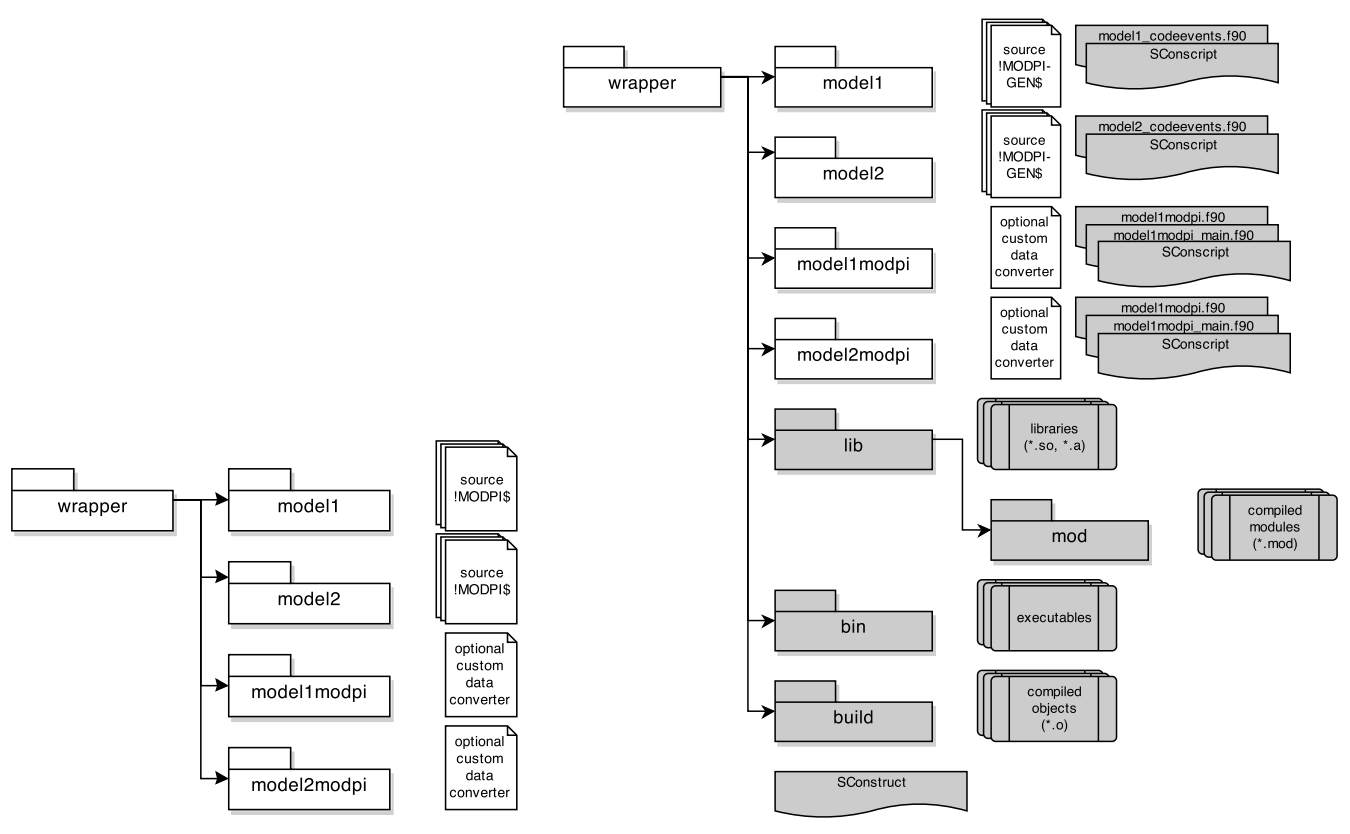

(a) Prior to code generation

(b) After code generation

Figure 6: Directory structure and naming conventions for automated MODPI wrapper generation. White elements are user-supplied, gray elements are generated. 
directory structure shown in Fig. 6.

Additional features of the code generator include options to remove all generated code, and perform automated execution of the integrated modeling system. Automated execution sets up and starts the MPI job in a fashion consistent with the model communication definitions found within the XML linkage file. The repository documentation discusses specific file locations, requirements and tools used for automatic code generation and execution of MODPI-compatible modeling systems.

\section{Case Study Application Linking DayCent and HYDRUS-1D}

To investigate invasiveness of MODPI, DayCent (Parton et al., 1998) and HYDRUS-1D (Simunek et al., 2008) are integrated using both MODPI and a previous approach where models were compiled into one executable and HYDRUS is called as a subroutine within DayCent (labeled the "subroutine approach" or "SUB"). DayCent provides daily simulation of biogeochemical fluxes using one-dimensional representation of vegetation, atmosphere, organic decomposition, and water movement through the soil (Parton et al., 2001). DayCent and HYDRUS-1D have been previously linked using the subroutine approach to analyze the added benefit of improved soil-water dynamics, because HYDRUS-1D provides necessary physically-based modeling of soil water content at fine time and spatial scales (Yuan et al., 2011). This linkage serves as a benchmark to compare the MODPI approach.

The subroutine approach is used to call the finite element solver within HYDRUS-1D as a subroutine within DayCent. Yuan et al. (2011) provided code for the implementation of the subroutine approach for DayCent- 
HYDRUS. Since DayCent and HYDRUS are compiled together, code bases of the integrated models are dependent on each other, and no longer stand as individual models. Additional changes to the code are difficult to separate from the integrated DayCent-HYDRUS modeling system. The original linkage combined an earlier version of DayCent with an earlier version of HYDRUS (Yuan et al., 2011). For our investigation, we utilized the current versions of DayCent and HYDRUS to integrate the models using the same approach and data conversion code. The updated linkage required nontrivial adaptation of the code to carefully preserve HYDRUS model state between daily timestep updates within DayCent. These characteristics and requirements demonstrate the difficulties of maintaining individual legacy model code to support integration with another model using the subroutine approach.

The second approach integrates the models using MODPI. DayCent and HYDRUS are minimally changed to compile as libraries. Original data conversion between DayCent and HYDRUS provided by Yuan et al. (2011) is preserved and supplied to the DayCent-HYDRUS model as a custom subscriber. By placing conversions within the wrapper program through custom subscribers, modifications to the DayCent library are avoided. MODPI directives are added to the original source files, and the MODPI wrapper code generator creates event constructs and event data within each model. The MODPI-compatible DayCent and HYDRUS executable files still execute independently, just as before MODPI integration. A wrapper program implements the MODPI interface for each model. The only dependency for MODPI-compatible DayCent and HYDRUS is the event library discussed in 
Section 4.1.

Fig. 7 illustrates the evolution of the DayCent model code base to make it compatible with MODPI. HYDRUS underwent a similar process. A connection between DayCent and HYDRUS using MODPI is achieved through the following three steps:

1. Minimal code modifications include creating a new program entry point, removing nonstandard model exits using the keyword stop, and refactoring code specific to the Windows operating system to compile and run HYDRUS on Linux using GNU compilers. ${ }^{5}$ Two MODPI directives are added to DayCent, and five to HYDRUS, in locations where the most desirable model states for linkage are achieved.

2. A single XML linkage file is built to provide specific information on data exchanges between the models.

3. Model events and a wrapper program are generated using the automated code generator.

The XML linkage file, events, and wrapper program for the DayCent-HYDRUS integrated model are found in the MODPI open-source repository. ${ }^{6}$ The link between DayCent and HYDRUS achieved through MODPI was intuitive because it provides access to model state at specific locations within the code. Unlike the subroutine approach, the MODPI-connected DayCent-HYDRUS did not require special consideration to preserve model state, because model

\footnotetext{
${ }^{5}$ Code for DayCent is available by email. Code for HYDRUS-1D is available in the repository https://bitbucket.org/adozier/fortmodpi.

${ }^{6}$ See footnote 3
} 


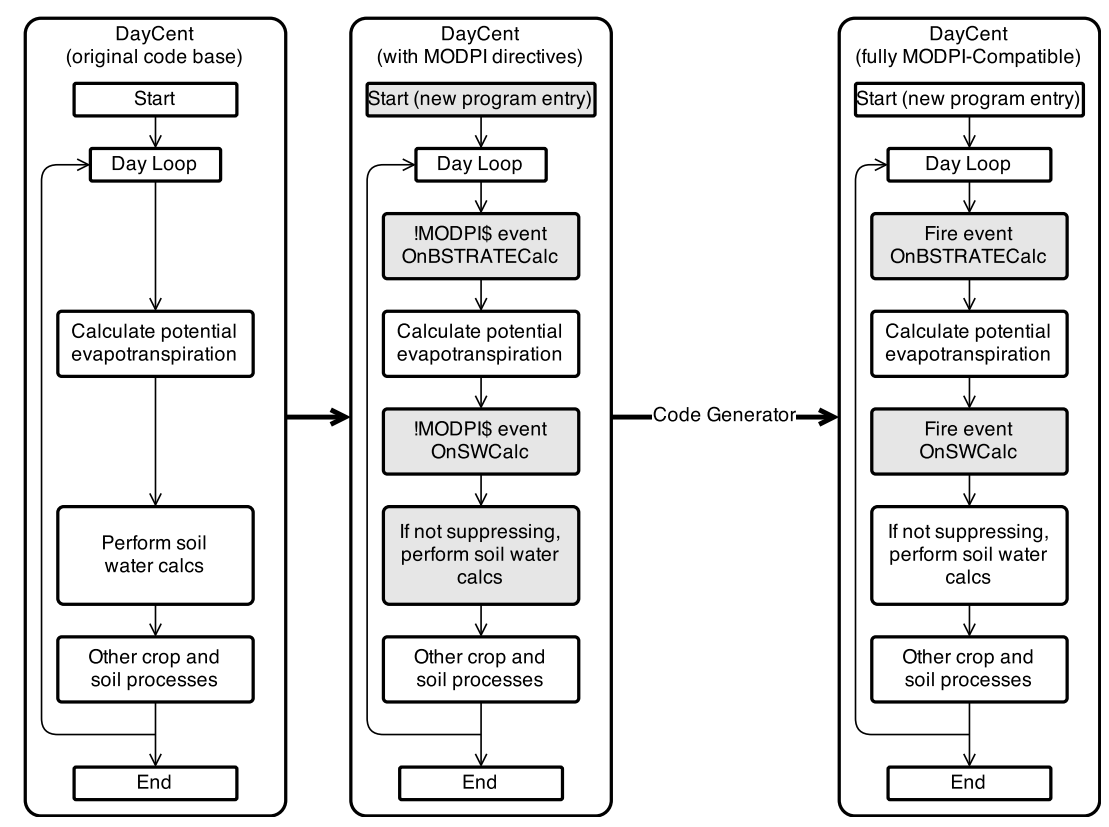

Figure 7: A flow chart displaying the evolution of DayCent from its original code base (left), to the MODPI-ready code (middle), to the full MODPI-compatible code (right). The MODPI integration platform generates events and event data that are placed into DayCent. Gray elements highlight incremental changes made within the legacy model code base.

state is internally preserved in HYDRUS when running as its own process without an adapted control flow.

Fig. 8 displays the flow of data for the DayCent-HYDRUS connection and the events at which custom data converters are subscribed to each model. At runtime, the wrapper programs for DayCent and HYDRUS are started as two separate processes, running in parallel. MODPI enables the models to exchange data using MPI over the network, or shared memory if both processes are running on one machine.

Within a DayCent-HYDRUS simulation, MODPI sends precipitation (pptsoil), 
potential evaporation (bserate), potential transpiration (bstrate), and root depth (tadep) from DayCent to HYDRUS prior to calculating soil water flux within HYDRUS. Since HYDRUS has an adaptable timestep but DayCent has a daily timestep, output data from HYDRUS is summarized at a daily timestep within the subroutine OnOutput_handler, in which the event OnCumQdlyCalc fires every day. Within the XML linkage file, the summarized daily output data are sent back to DayCent from the OnCumQdlyCalc event, not directly from the OnOutput event, which fires at a sub-daily timestep. The number of layers $\mathrm{n}$, the depth of each layer $\mathrm{x}$, an array cumqdly containing actual evaporation, transpiration, drainage, and runoff data, flux of water between soil layers vnacc, and soil water content of each layer thnew are received by DayCent at the event OnSwCalc. After arriving, the variables are converted within the custom subscriber convert_SWCalcs and used to update model state of DayCent. Other custom subscribers set variables or flags that aid linkage between DayCent and HYDRUS to improve console output readability.

\section{Results}

DayCent-HYDRUS serves as a case study to benchmark the MODPI integration platform presented in this paper. ${ }^{7}$ This section quantifies invasiveness and performance of MODPI in comparison with the subroutine (SUB) approach.

\footnotetext{
${ }^{7}$ Case study model files for the DayCent-HYDRUS integrated model are found at the Environmental Risk Assessment Management System (eRAMS) Resource Center: https: //erams.com/resources/Platform/MaaS/Model_Integration
} 


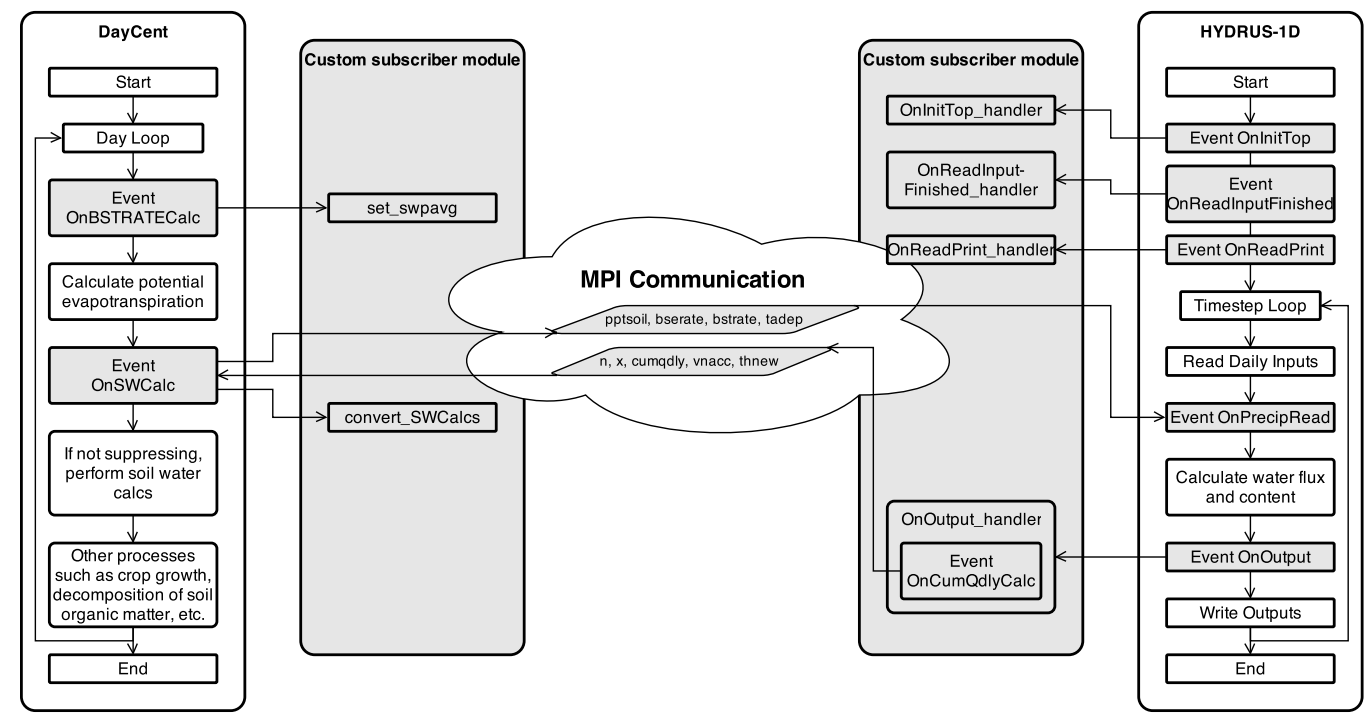

Figure 8: Data flow between MODPI-connected DayCent-HYDRUS. Links to and from events represent subscriptions that are automatically attained by the MODPI integration platform. Gray elements are essential for MODPI to perform linkage between DayCent and HYDRUS. Custom subscriber modules and the list of data to exchange between models found in the XML file are manually constructed, but events are automatically generated from MODPI directives within the code. 


\subsection{Code modifications}

The goal for developing MODPI is to reduce invasiveness of model integration tasks, thus necessitating use of metrics. Here, we quantify invasiveness using five software metrics based on lines of code (LOC):

1. Code modifications within each model code base (IN-LOC)

2. Code additions outside of each model code base (OUT-LOC)

3. Directive additions within each model (DIR-LOC)

4. Generated lines of code within the model (GEN-IN-LOC)

5. Generated lines of code outside of the model (GEN-OUT-LOC)

The first (IN-LOC) attempts to quantify the amount of work performed within each model code base, and serves as a surrogate measure for the amount of effort that would be required to maintain the integrated modeling system with changing versions of the original code base. The rest of the measures are specific to the coupled modeling system implementing MODPI in the case study. The second (OUT-LOC) attempts to measure the amount of work required to maintain the integrated model only when events or variables within the original model code base are renamed or moved. The third (DIR-LOC) attempts to quantify the amount of work performed to implement syntactically correct MODPI directives within the model code base, although there is no dependency of MODPI within the model through the use of directives since the directives are placed within the comments and not in code.

Finally, the GEN-IN-LOC and GEN-OUT-LOC metrics attempt to identify the amount of generated code that benefits abstraction and interoperability of model integration through inter-process communication. GEN-IN-LOC 
quantifies the number of lines of code to invoke events within the model, and is equal to 1 when global data is retrieved at an event and greater than 1 when using local data, which populates the event data construct. GEN-OUT-LOC quantifies the lines of code generated for the model wrapper that implements the Model Data Passing Interface for the model, but is compiled separately from the model code base.

The first three measures are better when lower assuming the same level of difficult, elegant code writing between each model integration technique. The fourth and fifth measures depend on modeler preference because they add value to the integrated modeling system but are additional lines of code not readily understood by the modeler. Therefore, GEN-IN-LOC and GENOUT-LOC are provided here for evaluation by the reader.

IN-LOC and DIR-LOC are invasive according to the definition in the introduction, and OUT-LOC and GEN-OUT-LOC is not since they are code additions outside of the original model code base. Attribution of invasiveness to GEN-IN-LOC is more subjective since some work is performed by the modeler run the generator, which is a relatively cheap operation compared to manually writing the code. MODPI outperforms SUB for code changes within the model (IN-LOC plus DIR-LOC), but requires more changes outside of the model (OUT-LOC) in addition to generated lines of code (GENIN-LOC and GEN-OUT-LOC).

Understand 3.1 (Scientific Toolworks, 2014) was used to quantify total LOC for each model, which do not include comments or blank lines. Code modifications within each model code base are identified as insertions and 
deletions in the Mercurial version control system. ${ }^{8}$ For each changeset in the Mercurial repository, all blank and commented lines were removed except for MODPI directives to quantify DIR-LOC. Although utilizing insertions and deletions through a version control system captures changed lines of code as an additional work item not otherwise captured when counting total LOC, changed lines of code are represented by both an inserted and deleted line of code, and are thus double-counted.

Table 1 shows code modifications necessary to integrate DayCent and HYDRUS for each approach. DayCent contains 236 source files with 23,057 LOC, and the original HYDRUS code base contains 9,034 LOC in 10 source files. The baseline HYDRUS code base used in this analysis is a Linuxcompatible version with 8,995 LOC. The SUB approach required 123 (and 562) LOC modifications to the DayCent model (and the HYDRUS-1D model, respectively) specific to the integrated modeling system. The MODPI approach required only 116 (and 204) LOC modifications within DayCent (and HYDRUS-1D), 11 (and 21) of which are manual modifications while the rest are generated. Total LOC modifications including the wrapper code to link DayCent (and HYDRUS-1D) using MODPI were 354 (and 515) LOC, while only a total of 87 (and 109) LOC were manually modified.

Five MODPI event directives and five import directives (10 DIR-LOC total) are added to the HYDRUS code. Import directives are required in FORTRAN for utilizing event constructs and subroutines found in modules. However, the same requirement does not apply to events invoked through

\footnotetext{
${ }^{8}$ Mercurial documentation and software is found at http://mercurial.selenic.com/.
} 
Table 1: Comparison of code modifications (insertions plus deletions) of lines of code (LOC) within DayCent and HYDRUS (Linux version) using the subroutine (SUB) and MODPI approaches to model integration.

\begin{tabular}{l|c|ccccc|c}
\hline \multicolumn{1}{c|}{ Code Base } & LOC & IN & OUT & DIR & GEN-IN & GEN-OUT & TOTAL \\
\hline DayCent & 23,057 & & & & & & \\
SUB & 23,157 & 123 & - & - & - & - & 123 \\
MODPI & 23,399 & 11 & 74 & 2 & 103 & 164 & 354 \\
\hline HYDRUS-1D & 8,995 & & & & & & \\
SUB & 9,066 & 562 & - & - & - & - & 562 \\
MODPI & 9,455 & 21 & 78 & 10 & 173 & 233 & 515 \\
\hline
\end{tabular}

subroutines from $\mathrm{C}$ source files, and thus only two MODPI event directives and zero import directives (2 DIR-LOC total) are added to the DayCent source code.

Yuan et al. (2011) provided 82 LOC that perform data conversion between HYDRUS and DayCent for the SUB approach within the DayCent model code, and is reported within the IN-LOC measure for DayCent. These LOC were adapted slightly to convert data within the MODPI linkage using a custom subscriber, and are therefore reported as OUT-LOC for DayCent (74 LOC) because the changes remain outside the original model code base. MODPI-compatible HYDRUS-1D incorporated 78 lines of converter code.

Filling event constructs with data and invoking them consume 103 and 173 LOC while generated wrapper codes include 164 and 233 LOC for DayCent and HYDRUS-1D, respectively. These generated LOC represent $75 \%$ and $79 \%$ of the 354 and 515 LOC changes for DayCent and HYDRUS-1D, respectively. These LOC are provided to the modeler as value-added attributes 
of MODPI, but they also represent code that is not readily understood by the modeler.

Minimal IN-LOC modifications for MODPI-compatibility come from adding flags to suppress redundant calculations and creating a new entry program (main) routine that calls the original entry as a subroutine. Total LOC for HYDRUS is based on a Linux version derived from the original Windows version with minimal changes. IN-LOC modifications for HYDRUS-1D are slightly disproportionate to the LOC within the SUB linkage (562 LOC) because several hundred file handle constants were changed to be able to compile HYDRUS-1D directly with DayCent.

All 123 (and 562) LOC modifications for the SUB approach in DayCent (and HYDRUS-1D) are integration-specific and consequently invasive. The MODPI approach does not require any integration-specific code changes within a model rendering it less invasive. However, the modeler can parameterize the automatic code generator to insert integration-specific event data to support a specific desired interface without effecting normal model execution or control flow. To illustrate this, modifications to DayCent include 12 lines of generated code that declare and allocate intermediate event data. This can be avoided by placing an event within the custom data converter (i.e., convert_SWCalcs in Fig. 8) that directly receives data from HYDRUS. To illustrate the minimally invasive potential for implementing MODPI, generated modifications to HYDRUS contain 0 integration-specific modifications. 


\subsection{Performance characteristics}

Use of MPI in the MODPI integration platform provides an opportunity to perform parallel processing on different CPUs and storage facilities, to potentially speed up model execution. The DayCent-HYDRUS integrated model, however, does not contain overlapping computations beyond initialization and output file writing, leading to little potential for parallelization. Consequently, network communication overhead may be an issue leading to slow model execution. To address this issue, four different HYDRUS models with differing discretizations of soil layers $(50,100,300$, and 1000) are used to help diagnose performance issues from arbitrarily increasing the number of computations between MPI network access. Computational overhead for DayCent-HYDRUS is assessed by comparing runtimes between the MODPIconnected integrated modeling system with those of the baseline subroutine (SUB) approach. Although runtime combines both overhead due to MODPI (and network communication) and time reduction due to parallelization, it serves to compare the SUB and MODPI approaches, and is more readily reproducible.

Tests are performed on a single SUN Blade x6270 server running Ubuntu 12.04 with two quad-core $2.8 \mathrm{GHz}$ Intel Xeon processors each with hyperthreading for a total of 16 logical cores. The servers offered 72 gigabytes of random access memory (RAM), and 145 gigabytes hard disk storage on a virtual local area network (VLAN) network connected with a 1 gigabit switch.

Table 2 summarizes runtimes of 200 identical test runs for each computational scenario, and Fig. 9 displays associated runtime variability. Overhead 


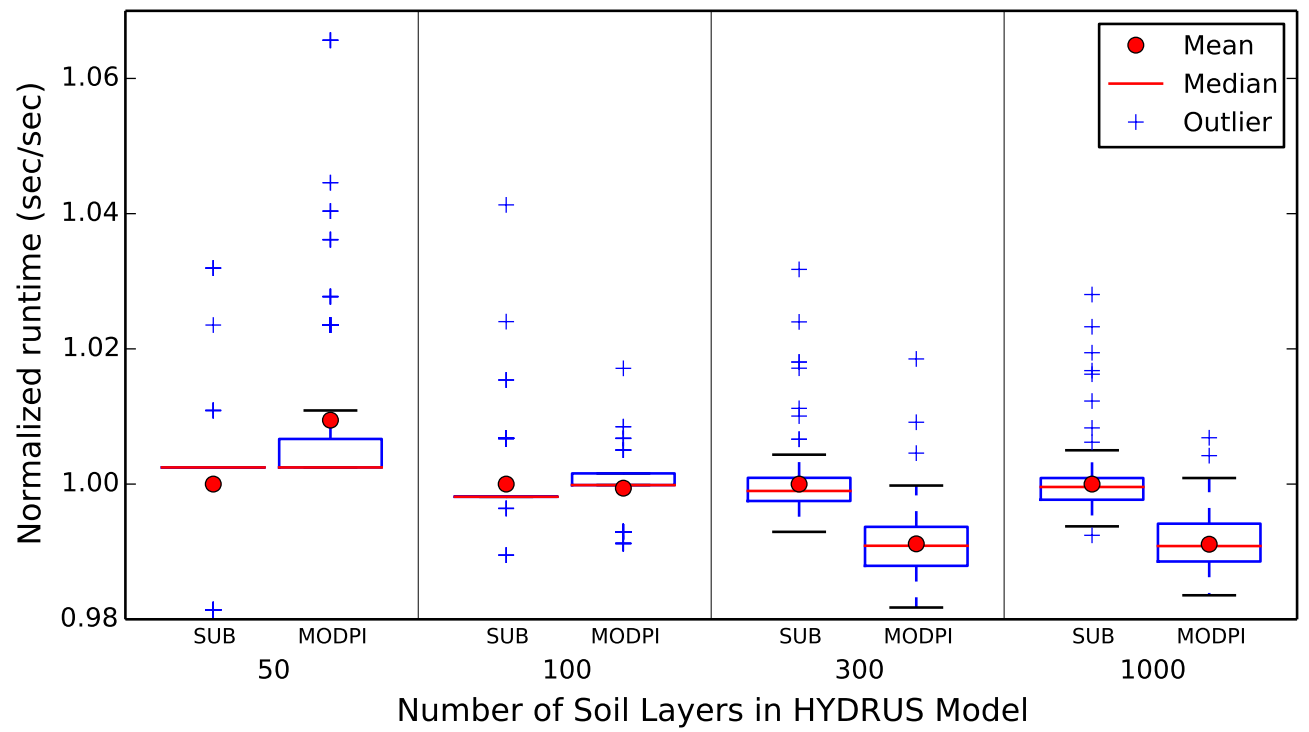

Figure 9: Normalized model runtimes of 200 identical simulations comparing the subroutine (SUB) approach with MODPI on a single machine with four different HYDRUS model setups to arbitrarily increase computations between network access. Runtime is normalized by the mean runtime of the SUB approach for each HYDRUS model setup. Outliers are runtimes that occur outside of the $95 \%$ confidence interval assuming a normal distribution. 
Table 2: Mean runtime (in seconds) for 200 DayCent-HYDRUS simulations when linked using both the subroutine (SUB) approach and MODPI. Four models were run with varying numbers of soil layers (50, 100, 300, and 1000) within HYDRUS to arbitrarily increase computation time between network access. Bold MODPI mean runtimes highlight statistically significant differences from the corresponding SUB mean runtime with a p-value $<$ 0.01 .

\begin{tabular}{c|c|c|c|c}
\hline \multirow{2}{*}{$\begin{array}{c}\text { Integration } \\
\text { Type }\end{array}$} & \multicolumn{4}{|c}{ HYDRUS Layers } \\
\cline { 2 - 5 } & $\mathbf{5 0}$ & $\mathbf{1 0 0}$ & $\mathbf{3 0 0}$ & $\mathbf{1 0 0 0}$ \\
\hline SUB & 2.374 & 5.791 & 43.79 & 75.53 \\
\hline MODPI & $\mathbf{2 . 3 9 7}$ & 5.787 & $\mathbf{4 3 . 4 0}$ & $\mathbf{7 4 . 8 6}$ \\
\hline
\end{tabular}

due to MPI network communication within MODPI-connected DayCentHYDRUS is minimal and is outweighed by benefits of parallelization for more computationally expensive scenarios. Mean runtime for the MODPIconnected model deviated from that of the SUB-connected model by $-0.9 \%$ to 0.9\%. MODPI outperforms SUB for computationally expensive models with 300 and 1000 soil layers. Additional performance analyses for MODPI found in Appendix A demonstrate larger overhead in virtualized environments and across machines, except in cases where there is little network communication relative to the number of computations and when running in Amazon EC2 instances.

\section{Discussion}

Very few code modifications are required to implement MODPI for a model compared to other model integration framework requirements for finegrained, multidirectional feedbacks that would generally require significant 
refactoring of the legacy model code base. For the case study, although code modifications remain very minimal for both the MODPI and SUB model integration approaches, MODPI is less invasive for the following reasons:

1. Code changes are not specific to the linkage between DayCent and HYDRUS

2. Code changes retain the control flow of the original code base

3. DayCent and HYDRUS are not compiled together, nor do they depend on each other or even on MODPI after integration

4. Fewer code changes are required within each model using MODPI than required by the SUB approach to model integration

By utilizing custom subscribers for external data manipulation and accessing variables and events by name, wrapper programs support a plug-and-play methodology for linking MODPI-compatible models or systems of models. Customized calculations can be turned on and off within the XML linkage file without having to recompile or generate model wrapper code again. Models using MODPI still retain model individuality and can be run individually or when connected to MODPI, and even run on separate operating systems and frameworks. These are benefits that could not be afforded even if the SUB approach was re-implemented with events to decouple the model code bases from the integrated modeling system as performed by (Wible, 2014).

There are advantages and disadvantages to using any model integration framework. Many situations exist where MODPI is a preferable approach to fine-grained model integration as discussed through the paper. However, there are situations when MODPI is not the preferable approach. Here we list situations on both sides of the spectrum starting with situations where 
MODPI is a preferable approach:

1. Integrating closed-source models with hidden or difficult input file formats

2. Performance overhead of input-output exchange between model runs is unacceptable

3. Subprocess representation within a model needs to be modified or overridden without changing the original model code

4. Poorly modularized legacy models that are otherwise difficult to integrate

5. Integrating models across different operating systems or frameworks

6. Integrating models without a predetermined number of connections to other models

Legacy models that are poorly modularized or that otherwise use a lot of global variables, which is characteristic of arguably most legacy models, are good use cases for MODPI because global variables grant MODPI easier access to model data. In this case, MODPI minimizes required refactoring for integration as compared to component-based frameworks, although care must still be taken to incorporate the correct model data in the linkage, as expected when using any model integration framework. Model integration with MODPI can also be performed across operating systems and has been shown to effectively link models across an Ubuntu and Windows systems. Although the current implementation of MODPI, the MODPI Integration Platform presented in this paper, has only been preliminarily tested in higher-level languages such as MATLAB and Java, the concept of multilingual frameworks is a well-known contribution of inter-process communication approaches. The 
use of events allows MODPI to grant publish-subscribe type interactions to models so that any number of subscribers (i.e., other models, visualizations, or analysis packages) can listen to events being invoked within the publishing model.

MODPI is not always the preferable approach to model integration due to the specific use cases that MODPI was designed to address. The following situations should prompt consideration before attempting to implement MODPI:

1. There is no intention to maintain up-to-date versions of integrated models and model codes are already easily interoperable

2. There is no need for within-simulation data access and exchange, or it is desirable to implement an iterative approach to obtain implicit solutions to equations

3. There is little potential for parallel execution but computational speed is high priority

4. The framework, language, or operating system is known not to support the inter-process communication mechanism (the MODPI Integration Platform currently supports both MPI and ZeroMQ)

If high performance is top priority for short jobs (less than 10 seconds), MODPI can help speed computations through parallel processing when possible, especially in high performance computing environments with high-end network hardware and proper configurations. Optimized configuration of a virtual environment and of the selected form of inter-process communication can also significantly improve performance. If no or very little parallel execution is possible for a specific model integration task, compiling mod- 
els together (when possible) or utilizing events to decouple the models (still within a single process) without overhead due to inter-process communication may be a preferable approach.

The MODPI integration platform potentially reduces the amount of programming work and the required level of programming expertise for specific model integration tasks through automated wrapper generation. However, there are a few tasks to be performed by the modeler that must be taken consideration. First, the modeler must conceptualize the model integration task correctly regarding both semantics and logistics as must be performed for integrating any model regardless of the framework or approach. Second, linkage locations within each model must be identified based on where certain data is available. Either events can be placed where the data is available, and custom subscribers can track references to the data, or data handling within the model is slightly refactored to provide certain data at a specific event. Third, the modeler must have knowledge of either event constructs, which is more probable with higher level languages, or the syntax of MODPI directives and rely on the MODPI code generation process to make a model compatible with MODPI. Fourth, nomenclature of the XML linkage files must be learned for specifying the data to be passed between models. Fifth, debugging a parallel application with automatically generated code may prove to be more difficult than sequential, manual approaches to model integration. Finally, custom data conversions will likely need to be performed (and maintained) by the modeler since MODPI does not currently require specific data structures within its interface. Aggregation and data conversion in space or time must currently be performed by the modeler, but this may change in future 
versions of MODPI. Benefits of custom data conversion includes flexibility, applicability to a broader set of model integration tasks, and computational speed addressed by removing potentially slower generic conversions.

One significant benefit is that MODPI can be used to integrate models with closed-source models in a fine-grained manner. The closed-source model developer must be willing to add events into the model, compile the model as a library with exported and relevant model data, and provide the names of events, event data, and other model data of interest to the modeler. In this manner, the model can still be used for integration with other models without having to share the source of the code or input file format. Closedsource model developers in the environmental sciences would benefit from this because they would not have to directly support interfaces of model integration platforms, but could still attract users that want to integrate the model with another. A finite set of interaction points within the closedsource model can be provided to users that would allow for varying degrees of granularity in integration and customization. This has been done in closedsource models like MODSIM (Labadie, 2010; Dozier, 2012) and FEFLOW (Becker and Schuttrumpf, 2011), for instance.

Potential users of MODPI include interdisciplinary researchers interested in model integration for improving process representation in geophysical modeling. Model developers for either open-source or closed-source models would be interested in using event constructs because they decouple the model from customized actions, other models, and model integration frameworks. Using events, model developers can maintain one code base instead of different code branches that implement interfaces for external integrated modeling systems. 
Modelers and consultants that perform integrated analysis of environmental systems may find MODPI a useful framework for minimizing programming time while allowing for fine-grained feedbacks between models.

\section{Conclusions}

The case study application demonstrates that minimal code modifications to a legacy model are required to implement MODPI for a model integration task. Only 11 and 21 lines of code were manually modified within legacy model code bases respectively, compared to 123 and 562 lines of manual code modifications for a baseline approach. At runtime, MODPI-compatible models incorporate 116 and 204 lines of code modifications, respectively, including generated code and MODPI directives found in the comments. Although the case study application required some user-written custom data converters and a bit of MODPI-generated wrapper code, MODPI requires less code changes in the original model code base than the baseline "subroutine" approach.

Performance tests demonstrate that MODPI not only has minimal overhead for the use case on a single machine, but provides speedup through parallel execution of linked models. Further evaluation of performance results as described in Appendix A highlights worsening overhead when running across two machines, except when little network communication is performed relative to computations or when running in virtual machines in Amazon EC2, depending on hardware, hypervisor, and virtual network setup. Mean runtime of MODPI-connected models varies between $-0.9 \%$ to $0.9 \%$ when compared to that of the subroutine approach, representing a shift from min- 
imal overhead to minimal speedup for a model integration scenario with few overlapping computations.

Inter-process communication provides MODPI a large degree of interoperability across languages, platforms, hardware, and license requirements, although only fully implemented and tested in Ubuntu and Windows with FORTRAN, C, and $\mathrm{C}++$. Closed-source models can be linked with other models using MODPI given that they come precompiled as a library with events. Customization allows any data structure to be converted and manipulated outside of the original model code base through use of event subscribers.

The design of MODPI attempts to address each of the six framework design targets discussed in Section 2. MODPI performs fine-grained, multidirectional feedbacks while remaining less invasive than other approaches, enhancing the maintainability and reusability of MODPI-integrated models. MODPI does not require any framework component or integration-specific code modification within the original model code base other than directives within model code comments and event constructs at runtime.

The MODPI-compatible model contains event constructs that generically provide read and write access to relevant data for model integration while preserving original control flow. The MODPI integration platform delivers most of these benefits by automated means, and therefore reduces modeler programming time.

Maintenance is minimal, but MODPI still does require care in event locations and naming, data access encapsulation and naming, and correct data conversion logic both within the wrapper program and the XML linkage file. 
Although inter-process communication offers interoperability and speed up through parallel execution, debugging a parallel program with automatically generated code is potentially more difficult than sequential approaches.

\section{Future Work}

Future work and developments will improve implementation of the MODPI Integration Platform to be more interoperable, generic, and widely applicable. Full MODPI implementations in Java, MATLAB, Python, R, and .NET languages will improve its assertion of interoperability. Although MODPI has already been tested on Ubuntu and Windows operating systems (and across both operating systems with one model on Ubuntu and one on Windows), more cross-platform tests for other operating systems such as Mac, Debian, BSD, HP-UX, Solaris, CentOS, and RHEL will increase its broader applicability.

MODPI benefits technically from not specifying data structures in order to allow model data to remain in its current structure to achieve minimal invasiveness, but this complicates generic data transformations in space, time, and across various data structures. Future research will focus on implementing generic data transformations within the MODPI Integration Platform followed by further advancements up the levels of conceptual interoperability model (Wang et al., 2009). Other future work on MODPI can be to implement it as a proxy between the model and a more generic model integration standard such as OpenMI or BMI for example, which will have a much broader impact on integrated modeling through use of open standards. Currently, MODPI does not detect potential deadlock situations, but 
could in the future. Since timesteps are a common feature of many legacy environmental models, MODPI could be improved to include a timesteptracking feature that detects when time periods are out of sync so that models do not hang at the end of execution waiting for more IPC. Another area of future work is to utilize MODPI to link more than two models or models in more spatial dimensions, since the current case study performs analysis only in one spatial dimension. Use cases for models with significant amounts of computation to be run in parallel will improve analysis of potential speedup benefits of MODPI.

\section{Acknowledgments}

This work is based upon work partially supported by a grant from the Agriculture and Food Research Initiative of the USDA National Institute of Food and Agriculture (NIFA), grant number \#2012-67003-19904, and partially by National Science Foundation IGERT Grant No. DGE-0966346: I-WATER: Integrated Water, Atmosphere, Ecosystem Education and Research Program at Colorado State University. The authors also acknowledge Dr. Fengming Yuan for providing the code that implemented a connection for DayCent-HYDRUS using the subroutine methodology.

\section{References}

Allan, B. A., Armstrong, R., Bernholdt, D. E., Bertrand, F., Chiu, K., Dahlgren, T. L., Damevski, K., Elwasif, W. R., Epperly, T. G. W., Govindaraju, M., Katz, D. S., Kohl, J. A., Krishnan, M., Kumfert, G., Larson, J. W., Lefantzi, S., Lewis, M. J., Malony, A. D., McInnes, L. C., Nieplocha, 
J., Norris, B., Parker, S. G., Ray, J., Shende, S., Windus, T. L., Zhou, S., May 2006. A Component Architecture for High-Performance Scientific Computing. Int. J. High Perform. Comput. Appl. 20 (2), 163-202. URL http://hpc.sagepub.com/cgi/doi/10.1177/1094342006064488

Argent, R. M., Mar. 2004. An overview of model integration for environmental applicationsâĂ ̌components, frameworks and semantics. Environ. Model. Softw. 19 (3), 219-234.

URL http://linkinghub.elsevier.com/retrieve/pii/ S1364815203001506

Armstrong, C. W., Ford, R. W., Riley, G. D., 2009. Coupling integrated earth system model components with BFG2. Concurr. Comput. Pract. Exp. 21 (6), 767-791.

Armstrong, R., Gannon, D., Geist, A., Keahey, K., Kohn, S., McInnes, L., Parker, S., Smolinski, B., 1999. Toward a Common Component Architecture for High-Performance Scientific Computing *. High Perform. Distrib. Comput., 115-124.

Armstrong, R., Kumfert, G., McInnes, L. C., Parker, S., Allan, B., Sottile, M., Epperly, T., Dahlgren, T., Feb. 2006. The CCA component model for high-performance scientific computing. Concurr. Comput. Pract. Exp. 18 (2), 215-229.

URL http://doi.wiley.com/10.1002/cpe.911

Baart, F., Ha, J., van Dam, A., Donchyts, G., Siemerink, M., 2014. Inter- 
active web-based flood modeling at country wide scale and planter size resolution. In: 7th Int. Congr. Env. Model. Softw. iEMSs, San Diego, CA.

Balaji, V., 2012. The Flexible Modeling System. In: Earth Syst. Model. - Vol. 3 Coupling Softw. Strateg. Vol. 3. Springer Science \& Business Media, pp. 33-41.

URL http://link. springer.com/10.1007/978-3-642-36464-8

Becker, B. P., Schuttrumpf, H., 2011. An OpenMI module for the groundwater flow simulation programme Feflow. J. Hydroinformatics 13 (1), 1-12.

Collins, N., Theurich, G., DeLuca, C., Suarez, M., Trayanov, A., Balaji, V., Li, P., Yang, W., Hill, C., da Silva, A., Aug. 2005. Design and Implementation of Components in the Earth System Modeling Framework. Int. J. High Perform. Comput. Appl. 19 (3), 341-350.

URL http://hpc.sagepub.com/cgi/doi/10.1177/1094342005056120

Dahlgren, T., Ebner, D., Epperly, T., Kumfert, G., Leek, J., Prantl, A., Jan. 2012. Babel UsersâĂŹ Guide. Tech. rep.

David, O., II, J. C. A., Lloyd, W. J., Green, T. R., Rojas, K. W., Leavesley, G. H., Ahuja, L. R., Ascough, J., Ascough II, J., Jan. 2013. A software engineering perspective on environmental modeling framework design: The Object Modeling System. Environ. Model. Softw. 39 (0), 201-213.

URL http://linkinghub.elsevier.com/retrieve/pii/ S1364815212000886http://www.sciencedirect.com/science/ article/pii/S1364815212000886 
David, O., Lloyd, W. J., Rojas, K. W., Arabi, M., Geter, F., Ascough, J. C., Green, T. R., Leavesley, G. H., Carlson, J. R., Jul. 2014. Model-as-aService (MaaS) using the Cloud Services Innovation Platform (CSIP). In: Ames, D. P., Quinn, N. W., Rizzoli, A. E. (Eds.), 7th Int. Congr. Env. Model. Softw. No. 1. International Environmental Modelling and Software Society (iEMSs), San Diego, CA.

Dean, J., 2009. Designs, Lessons and Advice from Building Large Distributed Systems. In: Keynote from LADIS. p. 73.

URL http://iepg.org/iepg/2009-11-ietf76/dean-keynoteladis2009.pdf

Donatelli, M., Rizzoli, A. E., 2008. A design for framework-independent model components of biophysical systems. In: iEMSs 2008 Int. Congr. Environ. Model. Softw. Vol. 2. iEMSs, Barcelona, Catalonia, pp. 727-734.

Dozier, A. Q., 2012. Integrated water and power modeling framework for renewable energy integration. Ms thesis, Colorado State University, Fort Collins, CO.

URL http://www.hydrofoundation.org/pdf/thesis/ DozierAndreThesis.pdf

Fredericks, J. W., Labadie, J. W., Altenhofen, J. M., 1998. Decision support system for conjunctive stream-aquifer management. J. Water Resour. Plan. Manag. 124, 69-78.

URL http://ascelibrary.org/doi/abs/10.1061/(ASCE)07339496(1998) $124: 2(69)$ 
Hill, C., DeLuca, C., Balaji, Suarez, M., Da Silva, A., 2004. The architecture of the earth system modeling framework. Comput. Sci. Eng. 6 (1), 18-28. URL http://scitation.aip.org/content/aip/journal/cise/6/1/ 10.1109/MCISE. 2004.1255817

Hintjens, P., 2013. ZeroMQ: Messaging for Many Applications, 1st Edition. O'Reilly Media, Inc.

Ibanez, E., Magee, T., Clement, M., Brinkman, G., Milligan, M., Zagona, E., Jul. 2014. Enhancing hydropower modeling in variable generation integration studies. Energy.

URL

http://linkinghub.elsevier.com/retrieve/pii/ S0360544214008391

Kim, N. W., Chung, I. M., Won, Y. S., Arnold, J. G., Jul. 2008. Development and application of the integrated SWATâĂŞMODFLOW model. J. Hydrol. 356 (1-2), 1-16.

URL http://linkinghub.elsevier.com/retrieve/pii/ S0022169408000991

Labadie, J. W., 2010. River Basin Management Decision Support System: User Manual and Documentation. Tech. rep., Fort Collins, CO.

URL http://modsim.engr.colostate.edu/version8.shtml

Laniak, G. F., Olchin, G., Goodall, J., Voinov, A. A., Hill, M., Glynn, P., Whelan, G., Geller, G., Quinn, N., Blind, M., Peckham, S., Reaney, S., Gaber, N., Kennedy, R., Hughes, A., Jan. 2013. Integrated environmental modeling: A vision and roadmap for the future. Environ. Model. Softw. 
39, 3-23.

URL http://linkinghub.elsevier.com/retrieve/pii/ $\mathrm{S} 1364815212002381$

Larson, J. W., Norris, B., Ong, E. T., Bernholdt, D. E., Drake, J. B., Elwasif, W. R., Ham, M. W., Rasmussen, C. E., Kumfert, G., Katz, D. S., Zhou, S., DeLuca, C., Collins, N. S., 2004. Components, the Common Component Architecture, and the Climate/Weather/Ocean Community. In: 84th Am. Meteorol. Soc. Annu. Meet.

URL http://Iibrary.cma.gov.cn:8087/ams\_data/AMS2004】_/ $69695 \cdot \operatorname{pdf}$

Lefantzi, S., Ray, J., Najm, H. N., 2003. Using the Common Component Architecture to Design High Performance Scientific Simulation Codes. In: Proc. Int. Parallel Distrib. Process. Symp. Vol. 00.

Lloyd, W. J., David, O., Ascough, J., Rojas, K., Carlson, J., Leavesley, G., Krause, P., Green, T., Ahuja, L., Oct. 2011. Environmental modeling framework invasiveness: Analysis and implications. Environ. Model. Softw. 26 (10), 1240-1250.

URL http://www.sciencedirect.com/science/article/pii/ S1364815211000867

Magnoni, L., 2015. Modern Messaging for Distributed Sytems. J. Phys. Conf. Ser. 608 (1), 12038.

URL http://stacks.iop.org/1742-6596/608/i=1/a=012038?key= crossref .43e2396affafe950cd4860a379e8d0bfhttp: //stacks . iop. org $/ 1742-6596 / 608 / i=1 / a=012038$ 
Matott, L. S., Babendreier, J. E., Purucker, S. T., Jun. 2009. Evaluating uncertainty in integrated environmental models: A review of concepts and tools. Water Resour. Res. 45 (6), n/a-n/a.

URL http://doi.wiley.com/10.1029/2008WR007301

Moore, R. V., Tindall, C. I., Jun. 2005. An overview of the open modelling interface and environment (the OpenMI). Environ. Sci. Policy 8 (3), 279-286.

URL http://linkinghub.elsevier.com/retrieve/pii/ S1462901105000456

Parton, W. J., Hartman, M., Ojima, D., Schimel, D., 1998. DAYCENT and its land surface submodel: Description and testing. Glob. Planet. Change 19 (1-4), 35-48.

URL http://dx.doi.org/10.1016/S0921-8181(98)00040-X

Parton, W. J., Holland, E. A., Del Grosso, S. J., Hartman, M. D., Martin, R. E., Mosier, A. R., Ojima, D. S., Schimel, D. S., 2001. Generalized model for $\mathrm{NO} x$ and $\mathrm{N} 2 \mathrm{O}$ emissions from soils. J. Geophys. Res. Atmos. 106 (D15), 17403-17419.

URL http://dx.doi.org/10.1029/2001JD900101

Peckham, S. D., Hutton, E. W., Norris, B., Apr. 2013. A component-based approach to integrated modeling in the geosciences: The design of CSDMS. Comput. Geosci. 53, 3-12.

URL http://linkinghub.elsevier.com/retrieve/pii/ S0098300412001252 
Salas, D., 2012. A Systematic Approach for Hydrological Model Couplings. Int'l J. Commun. Netw. Syst. Sci. 05 (06), 343-352.

URL http://www.scirp.org/journal/PaperDownload.aspx?DOI=10. 4236/ijcns. 2012.56045

Scientific Toolworks, 2014. Understand - Source Code Analysis and Metrics. URL http://www.scitools.com

Simunek, J., van Genuchten, M. T., Sejna, M., 2008. Development and Applications of the HYDRUS and STANMOD Software Packages and Related Codes. Vadose Zo. J. 7 (2), 587.

URL https://www. soils.org/publications/vzj/abstracts/7/2/587

The MPI Forum, 1993. MPI: Message Passing Interface.

URL http://citeseerx.ist.psu.edu/viewdoc/summary?doi=10.1.1. 52.5877

Valcke, S., Balaji, V., Craig, A., DeLuca, C., Dunlap, R., Ford, R. W., Jacob, R., Larson, J., O'Kuinghttons, R., Riley, G. D., Vertenstein, M., 2012. Coupling technologies for Earth System Modelling. Geosci. Model Dev. 5, 1589-1596.

Wang, W., Tolk, A., Wang, W., 2009. The Levels of Conceptual Interoperability Model: Applying Systems Engineering Principles to M\&S. SpringSim '09 Proc. 2009 Spring Simul. Multiconference (168), 9.

URL http://arxiv.org/abs/0908.0191

Wible, T., 2014. Enhanced watershed modeling and data analysis with a fully 
coupled hydrologic model and cloud-based flow analysis. Ph.D. thesis, Fort Collins, CO.

Yuan, F., Meixner, T., Fenn, M. E., Simunek, J., Sep. 2011. Impact of transient soil water simulation to estimated nitrogen leaching and emission at high- and low-deposition forest sites in Southern California. J. Geophys. Res. 116 (G3), G03040.

URL http://doi.wiley.com/10.1029/2011JG001644

\section{A. Appendix: Additional Performance Results}

Additional scenarios are assessed in this appendix to quantify potential computational overhead for a variety of machine setups. The DayCentHYDRUS model was executed on two different physical machines labeled "PM1" and "PM2" for operating systems running on "bare metal", and on two virtual machines labeled "VM1" and "VM2". These scenarios are compared with the MODPI-connected system straddling both machines, with DayCent on Machine 1 and HYDRUS on Machine 2 ("Both PMs" for physical machine cases, "Both VMs" for virtual machine cases). Due to increasing support for and usage of virtual machines in addition to interest in Modelingas-a-Service within the cloud (David et al., 2014), tests are executed in both a private cloud environment and within Amazon Elastic Compute Cloud (Amazon EC2) cloud platform, which help to assess performance in the presence of network jitter.

Machines in the private cloud environment are SUN Blade x6270 servers that run Eucalyptus 3.1 Infrastructure-as-a-Service (IaaS). Each machine 
has identical hardware with two quad-core $2.8 \mathrm{GHz}$ Intel Xeon processors each with hyper-threading for a total of 16 logical cores, 72 gigabytes of random access memory (RAM), 145 gigabytes hard disk storage, and use a virtual local area network (VLAN) network connected with a 1 gigabit switch. Virtual machines execute on a XEN 4.1.2 hypervisor, have 8 logical cores, 4 gigabytes RAM, and 30 gigabytes hard disk storage. The same network adapter is used for communication on both physical and virtual machine runs. For performance tests in Amazon EC2, we use the c3.xlarge virtual machine instances, which have one quad-core $2.8 \mathrm{GHz}$ Intel Xeon processor with 7.5 gigabytes of random access memory (RAM) and 40 gigabytes storage on a solid state drive. All machines in the analysis run Ubuntu 12.04 as the operating system.

By default, Open MPI utilizes shared memory when running on the same machine, but a large variance in performance occurs due to internal tuning options within the shared memory modules of Open MPI. To address this issue, we conduct tests restricting message passing communication to the Transmission Control Protocol (TCP), which gives more consistent performance results, and supports direct comparison as all tests then use TCP inherently.

Table A.1 displays mean runtime in seconds of 200 identical test runs for each model integration scenario, and Fig. A.1 summarizes runtime variability when comparing specific scenarios. Most observable differences in Table A.1 are statistically significant according to t-tests because of the large sample size and small variance. Many differences are very minuscule when normalized against the subroutine method on Machine 1 (SUB-M1) except for 
Table A.1: Mean runtime (in seconds) for 200 DayCent-HYDRUS test runs for both the subroutine integrated system (SUB) and the MODPI integrated system (MODPI) executed on both a physical and virtual machine in a private cloud (PM1 and VM1), in Amazon EC2 (Amazon VM1), and with DayCent on one machine and HYDRUS on another communicating over the network (Both PMs, Both VMs, and Amazon VMs). Four models were run with varying number of soil layers $(50,100,300$, and 1000) within HYDRUS to arbitrarily increase computation time between network access.

\begin{tabular}{c|l|c|c|c|c}
\hline \multirow{2}{*}{$\begin{array}{c}\text { Integration } \\
\text { Type }\end{array}$} & \multirow{2}{*}{ Machine } & \multicolumn{4}{|c}{ HYDRUS Layers } \\
\cline { 3 - 6 } & & $\mathbf{5 0}$ & $\mathbf{1 0 0}$ & $\mathbf{3 0 0}$ & $\mathbf{1 0 0 0}$ \\
\hline \multirow{4}{*}{ SUB } & PM1 & 2.4 & 5.8 & 43.8 & 75.5 \\
& VM1 & 2.4 & 5.8 & 43.8 & 75.7 \\
& Amazon VM1 & 2.1 & 5.1 & 38.5 & 66.1 \\
\hline \multirow{5}{*}{ MODPI } & PM1 & 2.4 & 5.8 & 43.4 & 74.9 \\
& AM1 & 2.4 & 5.9 & 44.5 & 76.7 \\
\cline { 2 - 6 } & Amazon VM1 & 2.3 & 5.5 & 41.4 & 71.2 \\
\hline & Both PMs & 2.9 & 6.3 & 44.2 & 75.7 \\
& Both VMs & 3.1 & 6.6 & 44.4 & 76.0 \\
& Amazon VMs & 2.7 & 5.6 & 38.4 & 65.6 \\
\hline
\end{tabular}

computationally inexpensive cases executed in the private cloud environment.

As discussed in the Results section, overhead due to MPI network communication when running on one machine within MODPI-connected DayCentHYDRUS is minimal and is outweighed by benefits of parallelization for the more computationally expensive scenarios when running on a physical machine. MPI network communication across machines in a private cloud has a much larger effect on runtime results. Increases in mean runtime when 


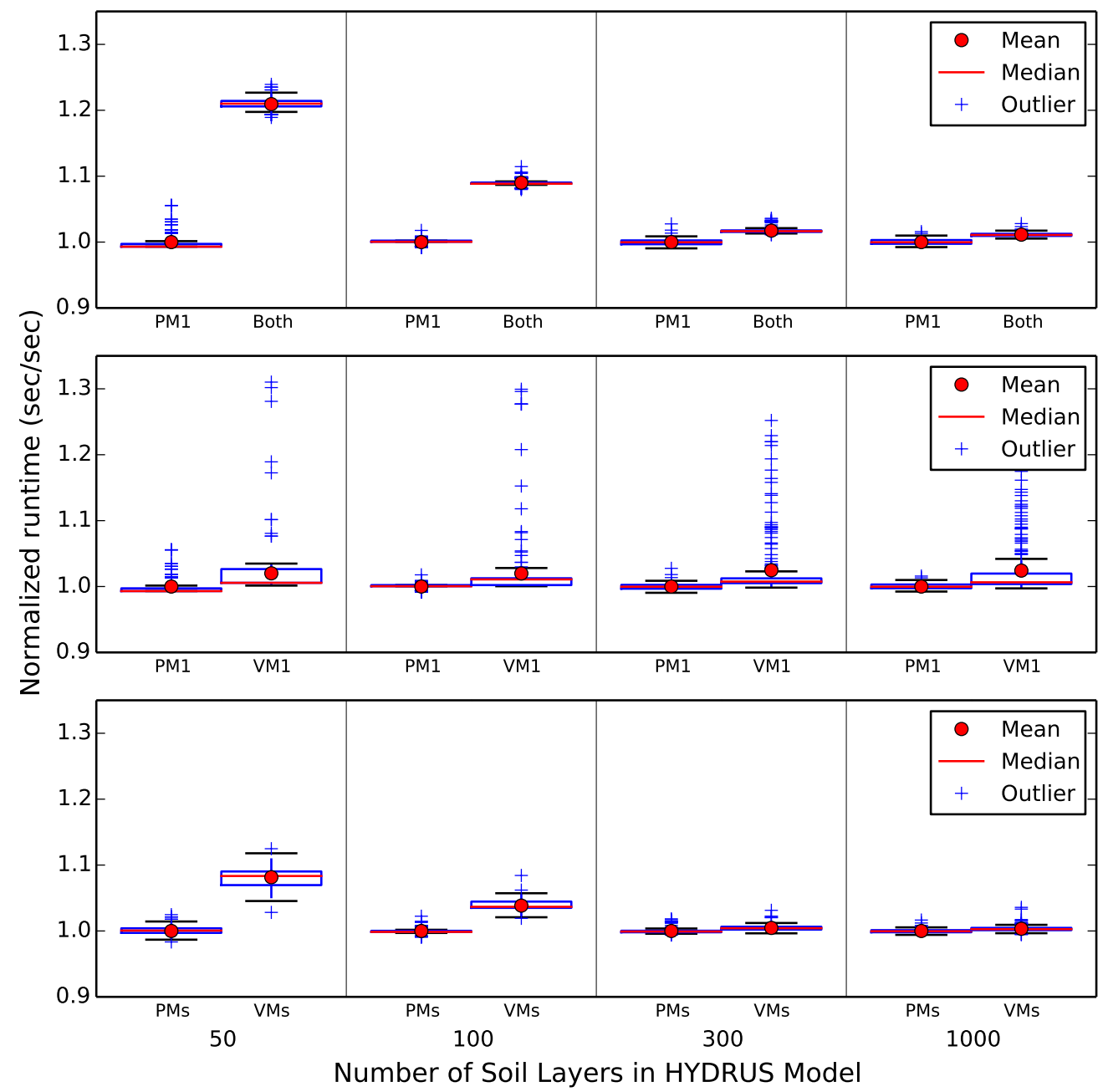

Figure A.1: Boxplots of normalized model runtime of 200 identical simulations on a private cloud comparing (top) a MODPI execution on a single physical machine and across two machines, (middle) a MODPI execution on a single physical machine and a single virtual machine, and (bottom) a MODPI execution across two physical machines with that across two virtual machines. Runtime is normalized by the mean runtime of the case on the left of the panel for each HYDRUS model setup (see mean runtimes in Table A.1). Outliers are runtimes that occur outside of the $95 \%$ confidence interval assuming a normal distribution. 
using the MODPI method on both physical machines (MODPI-Both-PMs) range from $1.1 \%$ to $20.9 \%$ as compared to the MODPI method running on one physical machine (MODPI-PM1) as shown in the top of Fig. A.1, but absolute differences in mean runtimes is relatively the same across scenarios, ranging from 0.5 to 0.8 seconds.

Overhead due to virtualization is minimal when on one machine, $0.1-0.5 \%$ more than baseline runtime for SUB scenarios and 2.0-2.5\% more than baseline for MODPI scenarios. As shown in the middle component of Fig. A.1, much more variation in runtime occurs for MODPI-connected models when running on virtual machines. The cost of virtualization is more exaggerated when running across two machines, increasing runtime by $0.3-8.2 \%$ when compared to MODPI running across two physical machines (see the bottom component in Fig. A.1) and by $1.5-30.8 \%$ when compared to MODPI performance on a single physical machine.

Performance results of scenarios executed within Amazon EC2 show a different pattern as summarized in Table A.2. MODPI executing on one virtual machine has approximately the same relative slowdown when compared with the SUB approach of $8 \%$ regardless of the number of computations. This may likely indicate a computational bottleneck due to scheduling of tasks in the virtualized environment instead of a network bottleneck, which would be expected to have the same absolute overhead in runtime. Operating across machines, MODPI has a relatively higher cost for jobs with little computational requirements with slowdown of 10.6-28.5\%, but little overhead for jobs with high computational requirements with speedup of $0.3-0.9 \%$. 
Table A.2: Increases in runtime (\%) for MODPI scenarios with two different machine setups within the private cloud and within Amazon EC2. The SUB-VM1 serves as the baseline scenario.

\begin{tabular}{c|l|ccccc}
\hline \multirow{2}{*}{ Platform } & \multirow{2}{*}{ Machine } & \multicolumn{4}{|c}{ HYDRUS Layers } \\
\cline { 3 - 6 } & & $\mathbf{5 0}$ & $\mathbf{1 0 0}$ & $\mathbf{3 0 0}$ & $\mathbf{1 0 0 0}$ \\
\hline \multirow{2}{*}{ Private cloud } & VM1 & $2.5 \%$ & $1.4 \%$ & $1.5 \%$ & $1.3 \%$ \\
& Both VMs & $31.4 \%$ & $12.5 \%$ & $1.2 \%$ & $0.4 \%$ \\
\hline \multirow{2}{*}{ Amazon EC2 } & VM1 & $8.7 \%$ & $7.9 \%$ & $7.5 \%$ & $7.6 \%$ \\
& Both VMs & $28.5 \%$ & $10.6 \%$ & $-0.3 \%$ & $-0.9 \%$ \\
\hline
\end{tabular}




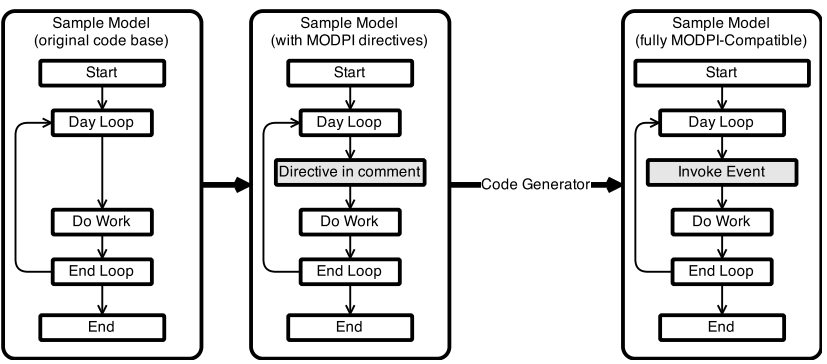

